Neurosurg Focus 27 (2):E3, 2009

\title{
Surgical anatomy and functional connectivity of the limbic system
}

\author{
Karl-Olof Lövblad, M.D., ${ }^{1}$ And Karl Schaller, M.D. ${ }^{2}$ \\ Departments of ${ }^{1}$ Diagnostic and Interventional Neuroradiology and ${ }^{2}$ Neurosurgery, Geneva University \\ Hospitals and Medical School, Geneva, Switzerland
}

\begin{abstract}
Object. The goal in this study was to highlight the potential of currently available imaging techniques for surgical planning of procedures in and around the limbic system.

Methods. The authors review traditional and newer imaging techniques as applied to neurosurgical planning. Today MR imaging techniques play a preponderant role. The various applications of functional techniques such as diffusion weighted, diffusion tensor, perfusion, and functional MR imaging methods are discussed.

Results. In addition to the high-resolution studies of anatomy that can be acquired, especially at higher field strengths ( $\geq 3 \mathrm{~T})$, MR imaging now also offers the possibility of acquiring functional, metabolic, hemodynamic, and molecular information on normal and pathological brain processes.

Conclusions. The knowledge obtained using the various imaging techniques contributes substantially to understanding the disease processes in a way that drastically improves surgical planning.

(DOI: 10.3171/2009.5.FOCUS09103)
\end{abstract}

KeY Words • limbic system • anatomy • magnetic resonance imaging

$\mathrm{D}$ UE to advances in neurosurgical operating techniques $^{28}$ over the past 2 decades and to have a better understanding of the neuropsychological processes involving the limbic ${ }^{21}$ and paralimbic ${ }^{2}$ systems, it has become necessary to make use of imaging tools that can on the one hand provide high-resolution 3D data sets and on the other hand also reflect functional anatomy, connectivity, and molecular metabolic processes. This is especially important if one considers the intrinsic gliomatous processes of these areas, which tend to spread along the underlying fibers. The introduction of higher-fieldstrength magnets and new imaging sequences allows the practitioner to improve anatomical, functional, and metabolic detail.

\section{The Limbic System}

The limbic system ${ }^{21}$ consists of the following elements, which are all directly or indirectly interconnected.

\section{The Hippocampus}

This structure ${ }^{7,27}$ is located in the mesial temporal lobe and represents the inferomedial wall of the temporal horn of the lateral ventricle. ${ }^{18}$ It can be especially well delineated on coronal FLAIR images. ${ }^{8}$ The head of the hip-

Abbreviations used in this paper: $\mathrm{DT}=$ diffusion tensor; $\mathrm{DW}=$ diffusion weighted; $\mathrm{fMR}=$ functional MR. pocampus is connected with the amygdala, which forms the anterosuperomedial roof of the tip of the temporal horn. Laterally, the collateral eminence marks the border to the parahippocampal gyrus: this represents the fold of the collateral sulcus (this sulcus can be seen from the inferior surface of the temporal lobe).

The hippocampal body and tail run along the brainstem, forming the lateral aspect of the ambient cistern. The hippocampal tail becomes thinner as it runs posteriorly, and supracallosally it becomes the indusium griseum, which then turns around the genu corporis callosi into the subcallosal region. The fimbriae of the hippocampus, as they move posteriorly along the choroidal fissure, become the crura of the fornix. The crura constitute the anterior and medial aspect of the anterior wall of the ventricular atrium, ${ }^{24}$ and move upward and join in the midline inferior to the splenium of the corpus callosum, and then run anteriorly and inferiorly to the mammillary bodies.

\section{The Parahippocampus}

This structure starts with the uncus, of which it represents the major portion, and thus represents the inferomedial surface of the temporal lobe. Mesially it also follows the brainstem and then runs cranially into the cingulum. The parahippocampal gyrus is continuous posteriorly with the lingual gyrus, and anterior and superiorly with the cingulate gyrus. 


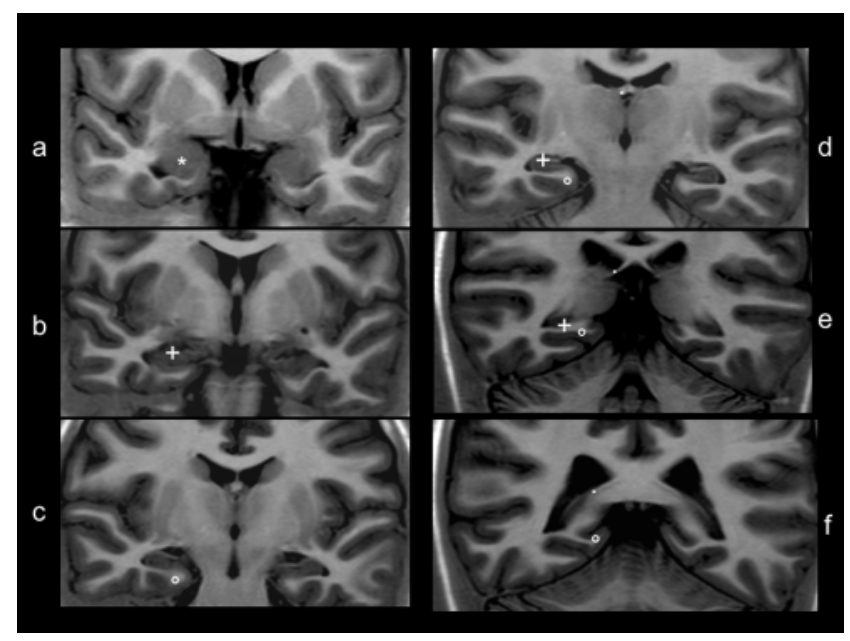

FIG. 1. Coronal T1-weighted MR imaging sections arranged in a progression from anterior to posterior, showing the various portions of the amygdala, the hippocampus, the parahippocampal gyrus, and the fornix. Structures are marked on the right side only. a: Amygdala (asterisk) forming the anterior and superior portion of the roof of the tip of the temporal horn of the lateral ventricle. b: The head of the hippocampus (plus). c: The uncal part of the parahippocampal gyrus (open circle). d: Body of the hippocampus (plus), the uncus (open circle), and the fornix (dot). e: The crus fornicis (dot), tail of the hippocampus (plus), and parahippocampal gyrus (open circle). f: The crus fornicis (dot) and the posterior part of the parahippocampal gyrus (open circle).

The cingulate gyrus moves around the corpus callosum, and then inferiorly and anteriorly it becomes the subcallosal area, which is part of the olfactory system. The subcallosal area is located where both hemispheres, via the septal area, connect on their frontomedial surfaces.

The limbic system connects via the entorhinal cortex and the uncinate fasciculus to the paralimbic system (that is, orbitofrontal cortex, temporopolar cortex, and insula).

These complex anatomical interconnections help explain the growth patterns and clinical symptomatology of tumors within the limbic and the paralimbic system, which may even cross over to the contralateral hemisphere via these fiber tracts.

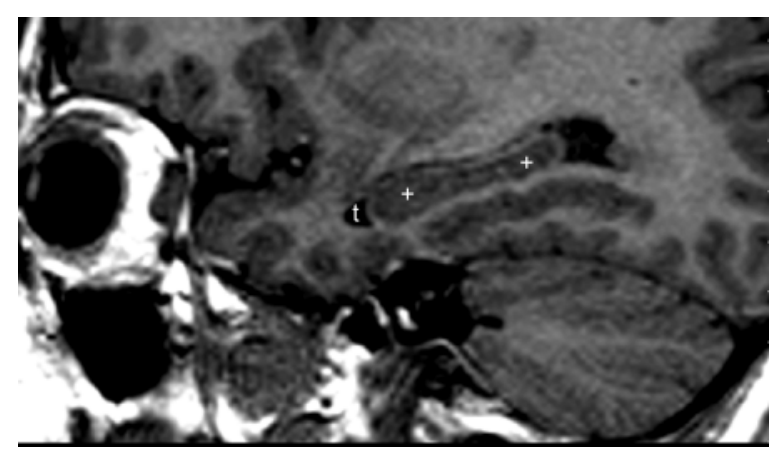

FIG. 2. Sagittal T1-weighted MR image of the temporal lobe: the tip of the temporal horn of the lateral ventricle $(t)$ and the head and body of the hippocampus (pluses) are shown.

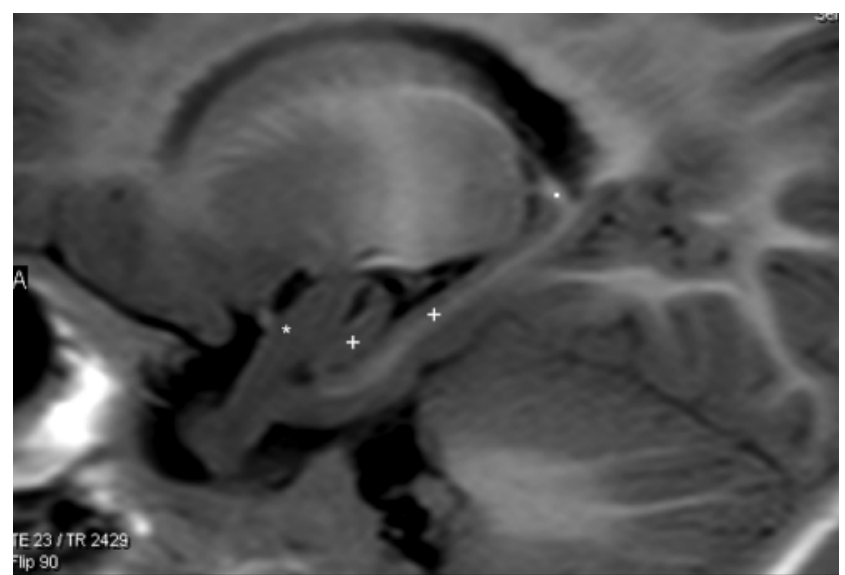

FIG. 3. Sagittal T1-weighted MR image of the temporal lobe, more mesial than Fig. 2: the crus fornicis (dot), head and fimbriae of hippocampus (pluses), and amygdala (asterisk) are shown.

\section{Imaging of the Limbic System}

\section{New MR Imaging Techniques}

Initially, MR imaging was able to acquire triplanar images of the brain with a high resolution (Figs. 1-4). The first machines could only provide these anatomical images at the cost of long acquisition times. The advent of fast imaging techniques such as echoplanar imaging 3,23 and high-field scanners $(\geq 3 \mathrm{~T})^{25,26}$ as well as parallel imaging techniques ${ }^{6}$ have now allowed improvements in both speed and resolution for most MR sequences. This also has allowed the revolution of fMR techniques, comprising the following: functional activation imaging, ${ }^{9} \mathrm{DW}$ imaging, ${ }^{14}$ DT imaging, ${ }^{15}$ perfusion imaging, ${ }^{19}$ spectroscopy, ${ }^{20}$ and molecular imaging techniques. ${ }^{10}$ Higher-fieldstrength MR units are slowly becoming the standard for advanced MR imaging; these units allow us to obtain images that have a stronger signal, and this will impact mainly T1 relaxation. This allows a gain in either resolution or speed. The techniques that will benefit from moving to higher fields are perfusion imaging, fMR imaging, and DT techniques. Another important technical development has been so-called parallel imaging, in which multiarray coils allow acquisition of many parts of the image at the same time, thus speeding up imaging while decreasing artifacts.

\section{The 3D Imaging Techniques}

These techniques are now available in both $\mathrm{T} 1$ and T2 weightings, and even FLAIR-weighted images can be acquired thus. This should allow us to obtain images for high-resolution purposes as well as for overlapping for neuronavigational purposes (Fig. 4). The MR imaging modality also has the advantage that it can directly acquire images in any chosen plane without having to resort to secondary reconstructions.

\section{Diffusion Imaging}

This modality ${ }^{14}$ is based on a modification of a simple spin echo sequence, in which 2 diffusion-sensitizing gradients are applied. These gradients apply motion to 


\section{Surgical anatomy and functional connectivity of the limbic system}

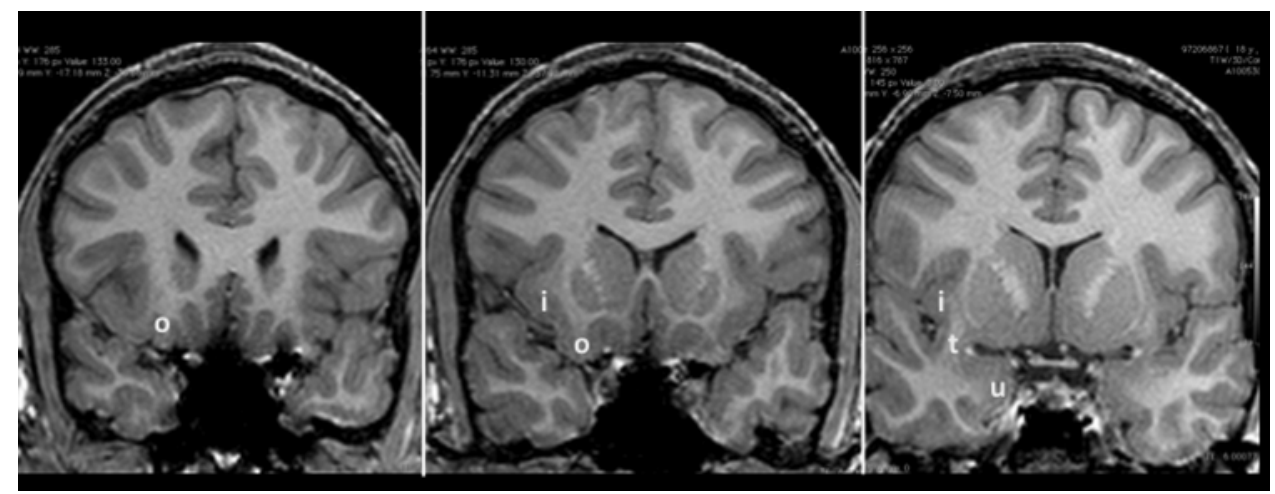

FIG. 4. Coronal T1-weighted sections arranged in a progression from anterior to posterior (left to right), showing the connection of the limbic and paralimbic systems, which explains the growth pattern of gliomas. Via these pathways, including the subcallosal area and anterior commissure, intrinsic tumors may even grow to the contralateral side. $\mathrm{i}=$ insular cortex; $0=0$ orbitofrontal gyrus; $\mathrm{t}$ = temporal stem; $\mathrm{u}=$ uncus.

the molecule on one hand, and on the other hand they extract the information about the motion in the short time between the gradients. This allows the physician to obtain information on the motility of the examined tissue. The more simple radiological use of this is DW imaging, which was applied to the study of stroke. One could see early changes corresponding to water redistribution but not its increase, ${ }^{17}$ which was reflected in a hypersignal and in a decrease in the apparent diffusion coefficient.

In the past few years, there has been growing interest in the value of DW imaging and eventual tissue characterization with the apparent diffusion coefficient in brain tumors. ${ }^{5}$ A further development was DT imaging, in which the gradients are applied in a higher number of directions $(>6)$, and we no longer obtain only a scalar but also a vector that represents water movement along white matter fibers. This can be either represented as maps of fractional anisotropy or reconstructed as white matter tracts (tractography). This allows us to explore connectivity between hemispheres and cortical areas (Fig. 5).
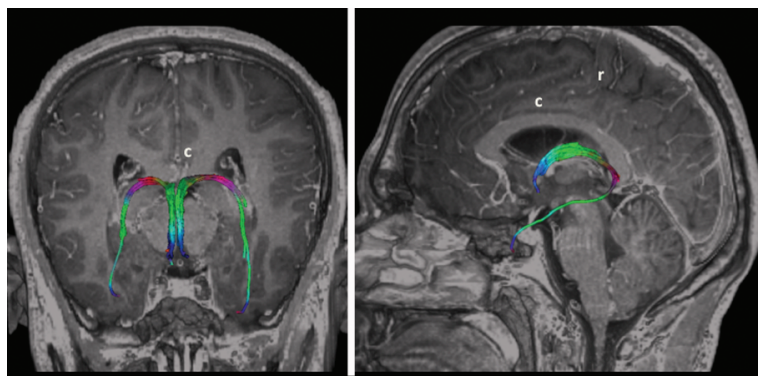

FIG. 5. Diffusion tractography of the fornix overlaid on a 3D T1weighted MR image in coronal (left) and sagittal (right) projections. The blue areas correspond to a craniocaudal direction of the fibers, the green areas to an anterior-posterior direction of the fibers, and the red areas to a left-right direction. The thin green portions correspond to the fimbriae of the hippocampus, which ultimately join the contralateral fibers at the level of the crus. The cingulum (c) runs above the corpus callosum and is connected to the postcentral region through the ramus circumflex (r). This is the reason why tumors essentially arising from the cingulum are sometimes confused with postcentral intrinsic tumors.

\section{Perfusion Imaging}

Perfusion studies with MR imaging can be obtained in many ways: with or without contrast as well as on T1 or T2* images. Most commonly, T2* imaging is performed, covering the whole brain, and a Gd chelate is injected intravenously, which causes an alteration in the magnetic field surrounding the vessel. This technique has been advocated for the follow-up of brain tumors to assess the beginning hyperperfusion that precedes or accompanies malignant transformation. ${ }^{12,13}$

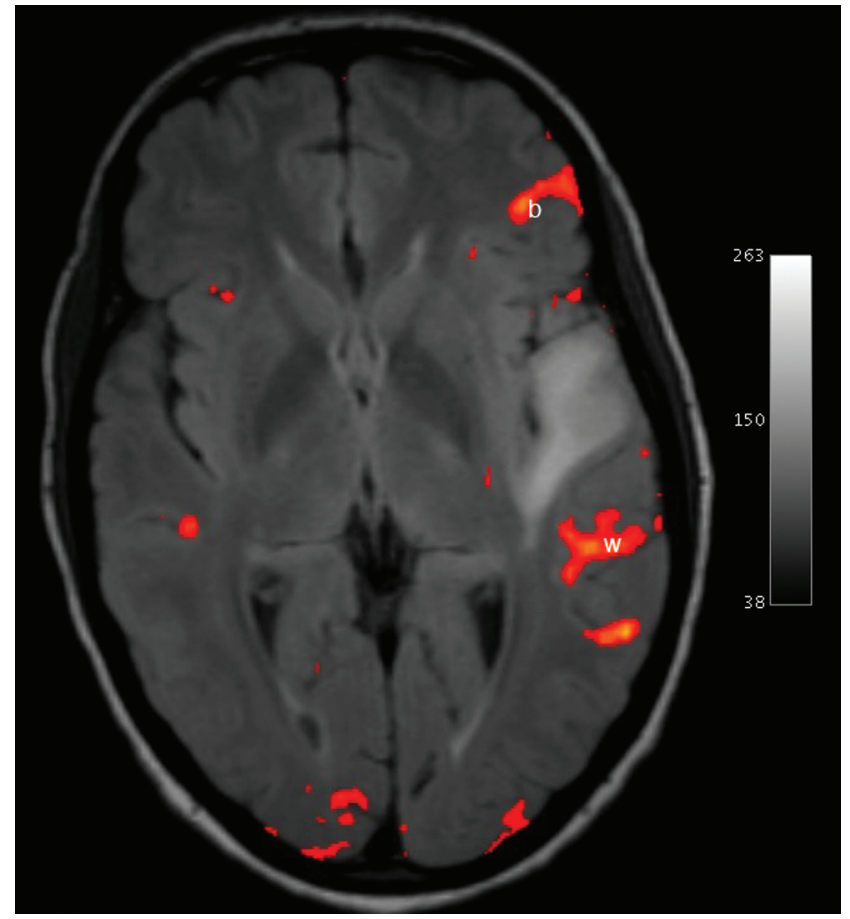

FIG. 6. An fMR imaging activation map overlaid on an axial FLAIR image showing a temporoopercular and insular tumor on the left, located at a distance from the activated language areas of Wernicke (w) and Broca (b). 


\section{K. O. Lövblad and K. Schaller}

Functional MR Imaging

This represents a further development of MR imaging technology; it relies on the uncoupling of metabolism and blood flow that occurs during activation related to function. This causes an increase in oxyhemoglobin (and thus a decrease of deoxyhemoglobin) on the venous side of the brain vasculature, which causes a change in the local magnetic field that will translate into an increase in signal on T2* images. This is called the blood oxygen level-dependent effect. The fMR imaging modality has been used widely both for clinical and research purposes to map brain function in a noninvasive way. Clinically, its most important impact has been in the presurgical mapping of anatomy in patients with brain tumors or lesional epilepsy (Fig. 6).

\section{Magnetic Resonance Spectroscopy}

This modality can provide in vivo visualization of the metabolic contents of the brain: this can be done either in a monovoxel or in a multivoxel way. Although it allows us to characterize the metabolic content of tumors, and can thus be used for the follow-up of lesions, ${ }^{11,22}$ caution is to be used when trying to assess the grade of single tumors at one time point.

\section{Susceptibility-Weighted Imaging}

Susceptibility-weighted imaging is a high-resolution imaging sequence that is very sensitive to alterations in local magnetic susceptibility. ${ }^{6}$ It can provide high-resolution images of the vessels and has pronounced signal decreases in areas where hemorrhage or calcifications are present; it can thus be used to detect small lesions such as cavernomas.

\section{Parameters Used for Imaging}

Very often, contrast media will be administered in patients with disordered brain processes to assess the integrity of the blood-brain barrier. ${ }^{16}$ In addition to the traditional contrast media based on Gd chelates, newer compounds are available, such as ultra small superparamagnetic iron oxide particles, which have allowed MR imaging to contribute to the new field of molecular imaging.

Images shown in this paper were acquired on a 3-T MR unit (Siemens Magnetom Treo) equipped with a head coil. Axial T2-weighted images (TE $101 \mathrm{msec}$, TR 4000 msec, 26 slices, 4-mm thickness, $372 \times 510$ matrix) and a sagittal T1-weighted multiplanar reconstruction sequence (TE $2.32 \mathrm{msec}$, TR $1900 \mathrm{msec}, 512 \times 512$ matrix) as well as a sagittal 3D FLAIR sequence (TE $420 \mathrm{msec}$, TR 6000 msec, $256 \times 256$ matrix, 162 1-mm-thick contiguous images) were obtained.

Diffusion imaging with a 30-directions scan was acquired as well, with b values of 1000 and 0 . Images were then postprocessed on a Leonardo workstation running the Siemens Neuro-3D software.

\section{Disclaimer}

The authors report no conflict of interest concerning the materials or methods used in this study or the findings specified in this paper.

\section{References}

1. Alimenti A, Delavelle J, Lazeyras F, Yilmaz H, Dietrich PY, de Tribolet N, et al: Monovoxel $1 \mathrm{H}$ magnetic resonance spectroscopy in the progression of gliomas. Eur Neurol 58:198209, 2007

2. Chen XZ, Jiang T, Li SW, Ai L, Dai JP: Dynamic radiological change of gliomas located in the paralimbic system and its clinical significance. Chin Med J (Engl) 121:713-715, 2008

3. Edelman RR, Wielopolski P, Schmitt F: Echo-planar MR imaging. Radiology 192:600-612, 1994

4. Guzman R, Altrichter S, El-Koussy M, Gralla J, Weis J, Barth A, et al: Contribution of the apparent diffusion coefficient in perilesional edema for the assessment of brain tumors. J Neuroradiol 35:224-229, 2008

5. Haacke EM, Xu Y, Cheng YC, Reichenbach JR: Susceptibility weighted imaging (SWI). Magn Reson Med 52:612-618, 2004

6. Heidemann RM, Seiberlich N, Griswold MA, Wohlfarth K, Krueger G, Jakob PM: Perspectives and limitations of parallel MR imaging at high field strengths. Neuroimaging Clin $\mathbf{N}$ Am 16:311-320, 2006

7. Henri M, Duvernoy JL, Vannson F, Cattin, Naidich TP: The Human Hippocampus: Functional Anatomy, Vascularization and Serial Sections with MRI. Heidelberg: Springer Verlag Berlin, 2004

8. Hui F, Cavazos JE, Tien RD: Hippocampus. Normal magnetic resonance imaging anatomy with volumetric studies. Neuroimaging Clin N Am 7:11-30, 1997

9. Kwong KK, Belliveau JW, Chesler DA, Goldberg IE, Weisskoff RM, Poncelet BP, et al: Dynamic magnetic resonance imaging of human brain activity during primary sensory stimulation. Proc Natl Acad Sci U S A 89:5675-5679, 1992

10. Lange N, Becker CD, Montet X: Molecular imaging in a (pre-) clinical context. Acta Gastroenterol Belg 71:308-317, 2008

11. Law M: MR spectroscopy of brain tumors. Top Magn Reson Imaging 15:291-313, 2004

12. Law M, Hamburger M, Johnson G, Inglese M, Londono A, Golfinos J, et al: Differentiating surgical from non-surgical lesions using perfusion MR imaging and proton MR spectroscopic imaging. Technol Cancer Res Treat 3:557-565, 2004

13. Law M, Young RJ, Babb JS, Peccerelli N, Chheang S, Gruber ML, et al: Gliomas: predicting time to progression or survival with cerebral blood volume measurements at dynamic susceptibility-weighted contrast-enhanced perfusion MR imaging. Radiology 247:490-498, 2008

14. Le Bihan D, Breton E, Lallemand D, Grenier P, Cabanis E, Laval-Jeantet M: MR Imaging of intravoxel incoherent motions: application to diffusion and perfusion in neurologic disorders. Radiology 161:401-407, 1986

15. Le Bihan D, Mangin JF, Poupon C, Clark CA, Pappata S, Molko N, et al: Diffusion tensor imaging: concepts and applications. J Magn Reson Imaging 13:534-546, 2001

16. Lövblad KO: Impact of contrast-enhanced CT and MRI on the management of patients with neurological diseases. Neuroradiol 49 (1 Suppl):S1-S2, 2007

17. Lövblad KO, Laubach HJ, Baird AE, Curtin F, Schlaug G, Edelman RR, et al: Clinical experience with diffusion-weighted MR in patients with acute stroke. AJNR Am J Neuroradiol 19:1061-1066, 1998

18. Rhoton AL Jr: Cranial anatomy and surgical approaches. Neurosurgery 53:1-746, 2003 


\section{Surgical anatomy and functional connectivity of the limbic system}

19. Rosen BR, Belliveau JW, Vevea JM, Brady TJ: Perfusion imaging with NMR contrast agents. Magn Reson Med 14:249265, 1990

20. Ross B, Kreis R, Ernst T: Clinical tools for the 90s: magnetic resonance spectroscopy and metabolite imaging. Eur J Radiol 14:128-140, 1992

21. Sitoh YY, Tien RD: The limbic system. An overview of the anatomy and its development. Neuroimaging Clin N Am 7:1-10, 1997

22. Soares DP, Law M: Magnetic resonance spectroscopy of the brain: review of metabolites and clinical applications. Clin Radiol 64:12-21, 2009

23. Stehling MK, Turner R, Mansfield P: Echo-planar imaging: magnetic resonance imaging in a fraction of a second. Science 254:43-50, 1991

24. Timurkaynak E, Rhoton AL Jr, Barry M: Microsurgical anatomy and operative approaches to the lateral ventricles. Neurosurgery 19:685-723, 1986

25. Vargas MI, Delavelle J, Kohler R, Becker CD, Lovblad K: Brain and spine MRI artifacts at 3 Tesla. J Neuroradiol [epub ahead of print], 2008
26. Willinek WA, Kuhl CK: 3.0 T neuroimaging: technical considerations and clinical applications. Neuroimaging Clin $\mathbf{N}$ Am 16:217-228, 2006

27. Wen HT, Rhoton AL Jr, de Oliveira E, Cardoso AC, Tedeschi $\mathrm{H}$, Baccanelli M, et al: Microsurgical anatomy of the temporal lobe: part 1: mesial temporal lobe anatomy and its vascular relationships as applied to amygdalohippocampectomy. Neurosurgery 45:549-592, 1999

28. Yaşargil MG, Teddy PJ, Roth P: Selective amygdalo-hippocampectomy. Operative anatomy and surgical technique. Adv Tech Stand Neurosurg 12:93-123, 1985

Manuscript submitted April 14, 2009.

Accepted May 19, 2009.

Address correspondence to: Karl-Olof Lövblad, M.D., Service Neuro-diagnostique et Neuro-Interventionnel, DISIM, Hôpitaux Universitaires de Genève, 24 rue Micheli-du-Crest, 1211 Genève 4, Swizerland. email: karl-olof.lovblad@hcuge.ch. 